\title{
Eleven years of Maraviroc experience and limited side effects in a HIV-1 experienced patient. Long term antiretroviral observation
}

\author{
Brescini L ${ }^{1}$, Weimer LE ${ }^{2 *}$, O Cirioni ${ }^{1}$, G Morroni $^{1}$ and Giacometti $\mathrm{A}^{1}$ \\ ${ }^{1}$ Institute of Infectious Diseases and Public Health, Università Politecnica delle Marche, Ancona, Italy \\ ${ }^{2}$ Centre of Global Health, Infectious Diseases, Istituto Superiore di Sanità, Rome, Italy
}

\begin{abstract}
Maraviroc, originally designated UK-427857, is a small molecule and the first oral antiretroviral drug in the CCR5 receptor antagonist class used in the treatment of HIV infection. This drug classed as an entry inhibitor, was developed by the drug company Pfizer in its UK labs located in Sandwich. On April 24, 2007 the U.S. Food and Drug Administration advisory panel reviewing maraviroc's New Drug Application unanimously recommended approval for the new drug, and the drug received full FDA approval on August 6, 2007 for use in treatment experienced patients. Maraviroc is extensively metabolized by CYP3A4, with renal clearance accounting for approximately $23 \%$ of total clearance and has been shown to achieve an undetectable HIV-1 RNA level in clinically advanced, class three antiretroviral treatmentexperienced adults with evidence of CCR5-tropic HIV-1 replication despite ongoing antiretroviral therapy. It is well tolerated, and its development is responding to a desperate need for new classes of antiretroviral agents that can target novel steps of the HIV lifecycle and do not share cross resistance with currently available therapy. This CCR5 receptor antagonist reviews clinically relevant pharmacological, long term therapeutic efficacy.
\end{abstract}

Our case report aims to explain the impact of Maraviroc co-administered with agents from all classes of antiretroviral therapy in a HIV-1 experienced patient along eleven years of antiretroviral experience.

\section{Background}

Maraviroc, is a small molecule and the first oral antiretroviral drug in the CCR5 receptor antagonist class used in the treatment of HIV infection and it has proven potent efficacy in treatmentexperienced patients with multiple drug failure.

This drug classed as an entry inhibitor, was developed by the drug company Pfizer in its UK labs located in Sandwich.

On April 24, 2007 the U.S. Food and Drug Administration advisory panel reviewing maraviroc's New Drug Application unanimously recommended approval for the new drug, and the drug received full FDA approval on August 6, 2007 for use in treatment experienced patients [1]. Maraviroc is extensively metabolized by CYP3A4, with renal clearance accounting for approximately $23 \%$ of total clearance and has been shown to achieve an undetectable HIV-1 RNA level in clinically advanced, class three antiretroviral treatment-experienced adults with evidence of CCR5-tropic HIV-1 replication despite ongoing antiretroviral therapy. It is well tolerated, and its development is responding to a desperate need for new classes of antiretroviral agents that can target novel steps of the HIV lifecycle and do not share cross-resistance with currently available therapy [2]. This CCR5 receptor antagonist reviews clinically relevant pharmacological, long term therapeutic efficacy. Our case report aims to explain the impact of Maraviroc co-administered with agents from all classes of antiretroviral therapy in a HIV-1 experienced patient along eleven years of antiretroviral experience.

\section{Case description}

Our patient, female with HIV-1 infection diagnosed in September 1989, heterosexual 46-year-old Italian, presenting in the Hospital of Ancona (January 2008) without coinfections and at baseline CD4 count of 116 cells/ $\mu$ l, HIV-RNA $2604 \mathrm{cp} / \mathrm{ml}$ (detection limit 50 copies/ $\mathrm{ml}$ ). In January 2008, she started Maraviroc, zidovudine, lamivudine, darunavir, ritonavir, attended clinic regularly and reported good treatment adherence.

Her multidrug-resistance profile at baseline is (test TRUGENE HIV-1): reverse transcriptase mutations M41L, K65R, K70R, V75I, F77L, Q151M; protease inhibitor mutations L10V, K20R, L33F, M36I, M46I, G48V, I54T, L63P, A71V, V77I, V82I, I84V.

At time of admission in our Department of Infectious Diseases laboratory analysis are: AST $19 \mathrm{IU} / \mathrm{ml}$ (reference range, 1-36 IU/l), ALT $23 \mathrm{IU} / \mathrm{ml}$ (reference range, 1-36 IU/l), total bilirubin 0,4 mg/dl , triglycerides $152 \mathrm{mg} / \mathrm{dl}$, total cholesterol $269 \mathrm{mg} / \mathrm{dl}$, HDL cholesterol $59 \mathrm{mg} / \mathrm{dl}$, creatinine $0,70 \mathrm{mg} / \mathrm{dl}$., glycemia $82 \mathrm{mg} / \mathrm{dl}$ without history of insulin resistance., hypocomplementemia, glomerulonephritis,

*Correspondence to: Liliana Elena Weimer, Centre of Global Health, Infectious Diseases, Istituto Superiore di Sanità, Rome, Italy, Tel: 390649903228; Fax: 390649387199; E-mail: liliana.weimer@iss.it

Key words: HIV-1 infection, long-term maraviroc observation, limited side effect

Received: February 06, 2019; Accepted: February 19, 2019; Published: February 22,2019 
and autoimmune disorders and lipodystrophy. After two months of therapy the patient reveals (at objective examination) a mixed lipodystrophy. In April 2008 she switched to AZT/3TC, which was modified to tenofovir and emtricitabine for drugs intolerance. During subsequent follow up our patient has been maintaining good clinical conditions and a discrete adherence to HAART regimen and limit side effects.

In May 2010 biochemical , hematological and viro immunological parameters have demonstrated a good response to Maraviroc without intolerance, drugs resistance and side effects: CD4 T-cell count of 442 cell $/ \mathrm{mm} 3$ and undetectable plasma HIV RNA concentration (detection limit 50 copies $/ \mathrm{ml}$ ), total bilirubin $0,50 \mathrm{mg} / \mathrm{dl}$, triglycerides $153 \mathrm{mg} /$ $\mathrm{dl}$, total cholesterol $271 \mathrm{mg} / \mathrm{dl}$, HDL cholesterol $52 \mathrm{mg} / \mathrm{dl}$, creatinine $0,80 \mathrm{mg}$., glycemia $73 \mathrm{mg} / \mathrm{dl}$ ALT concentration to $13 \mathrm{IU} / \mathrm{l}$ and AST 20 IU/1 (reference range, 1-36 IU/l) and objective signs of lipodystrophy have decreased.

In July 2017 our patient switched PRESISTA (Darunavir/ritonavir) to REZOSTA (Darunavir $800 \mathrm{mg}+$ Cobicistat $150 \mathrm{mg}$ ) for antiviral simplification.

In July 2018 she switchet TRUVADA (TDF+FTC) to DISCOVY (TENOFOVIR ALAFENAMIDE/EMTRICITABILE) for low compliance. Currently, after eleven years of therapy with Maraviroc, tenofovir, emtricitabine, ritonavir, darunavir, cobicistat laboratory examination and objective examination reveals a very important reduction signs of mixed lipodystrophy without any interruption of antiretroviral therapy containing protease inhibitors as backbone (Figures 1 and 2).

In January 2019: CD4 T-cell count of 626 cell $/ \mathrm{mm} 3$ and undetectable plasma HIV RNA concentration (detection limit 50 copies $/ \mathrm{ml}$ ), AST 17 $\mathrm{IU} / \mathrm{ml}$, ALT $19 \mathrm{IU} / \mathrm{ml}$, total bilirubin 0,5 mg/dl, triglycerides $132 \mathrm{mg} / \mathrm{dl}$, total cholesterol $200 \mathrm{mg} / \mathrm{dl}$, HDL cholesterol $72 \mathrm{mg} / \mathrm{dl}$, creatinine 0,85 mg., glycemia $81 \mathrm{mg} / \mathrm{dl}$.

Surgery (excision or liposuction) has not been performed on our patient because his severe fat accumulation was reduced without the interruption of protease inhibitors.

This case demonstrates that MARAVIROC in combination with DARUNAVIR/COBICISTAT (REZOLSTA $800 \mathrm{mg} / 150 \mathrm{mg}$ film-coated tablets) and TENOFOVIR ALAFENAMIDE /EMTRICITABINE (DESCOVY $200 \mathrm{mg} / 10 \mathrm{mg}$ ) was effective in suppressing the viral load of a highly treatment experienced patient with HIV-1.

In treatment experienced and multidrug resistance patients with HIV-1 infection, MARAVIROC has been shown to have maximum benefit when introduced with at least one other active new antiviral agent and has been observed a limit side effect.

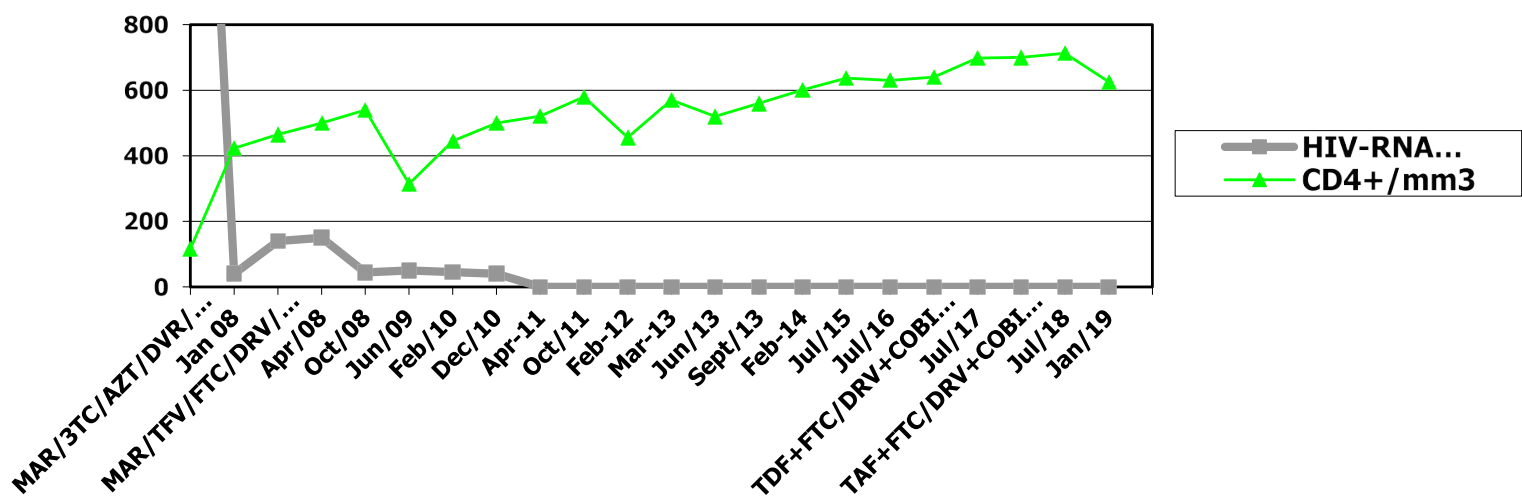

Figure 1. HIV-RNA viral load, CD4+ cell count and antiretroviral treatment

MAR: Maraviroc; COBI: Cobicistat; TAF: Tenofovir alafenamide; AZT: Zidovudine; TFV: Tenofovir; FTC: Emtricitabina; DAV: Darunavir; RTV: Ritonavir; COBI: Cobicistad; 3TC: Lamivudine; DRV: Darunavir

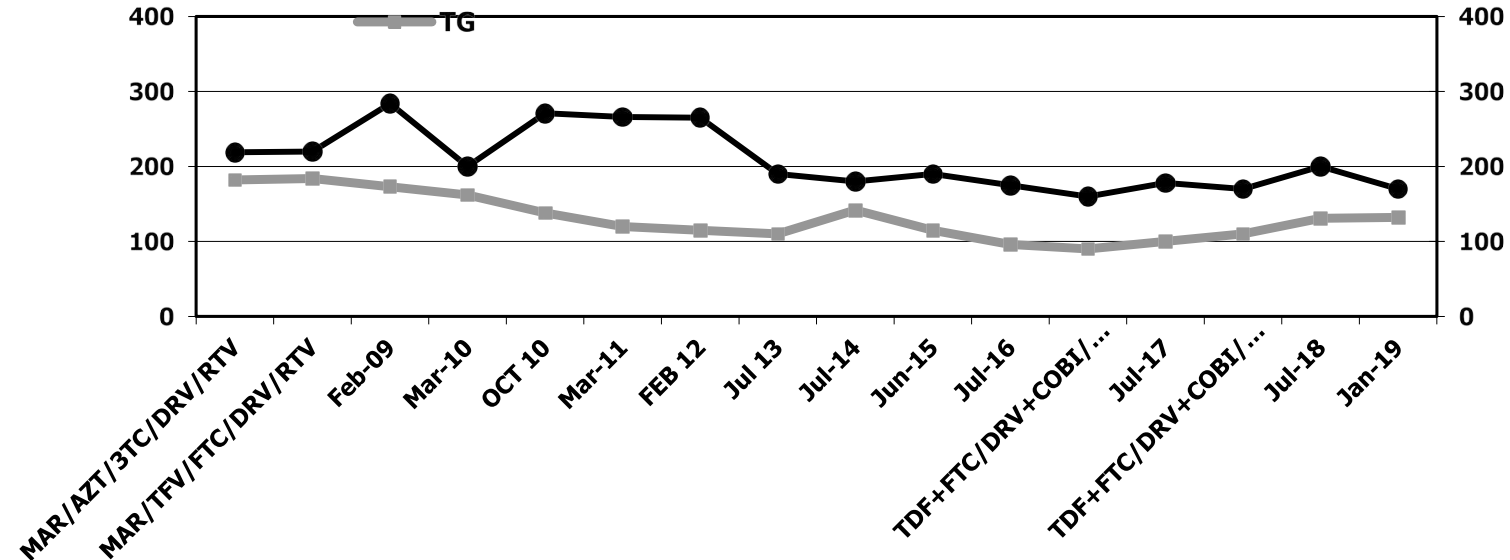

Figure 2. Serum transaminases levels

MAR: Maraviroc; COBI: Cobicistat; TAF: Tenofovir alafenamide; AZT: Zidovudine; TFV: Tenofovir; FTC: Emtricitabina; DAV: Darunavir; RTV: Ritonavir; COBI: Cobicistad; 3TC: Lamivudine 


\section{Discussion}

Eradication of HIV infection cannot be archived with existing regimens. The goals of therapy are the prolonged suppression of viral levels to less than detection limits $(<50$ copies $/ \mathrm{mL}$ for Amplicor assay, $<75$ copies/ $\mathrm{mL}$ for VERSANT assay, and $<80$ copies $/ \mathrm{mL}$ for Nuclei Sens assay), with the aim to restore and preserve immunologic function, improve quality of life, and avoid HIV-associated morbidity and mortality. Treatment success needs strict lifelong drug adherence and Maraviroc monotherapy has a high potency and long half-life, allowing single-pill dosing.

Maraviroc is only effective against CCR5-tropic virus, which predominates throughout infection but is more common in patients at the early asymptomatic stage of infection. It is not known how quickly resistance may develop to maraviroc in clinical practice. Current evidence supports the continued development of maraviroc as a potentially useful, alternative treatment for the management of HIV infection

Our patient, experienced and multidrug resistant, has never stopped Maraviroc along six years of antiretroviral therapy and therefore has not developed any resistance.

Preliminary evidence indicates that maraviroc is likely to provide an alternative therapy for treatment-experienced patients, and for treatment-naive patients who are newly infected with drug-resistant virus. However, improvements in efficacy or short- and long-term side effects for maraviroc compared with currently available regimens in treatment-naïve patients could positively impact on it use in this patient population provided that its use does not promote the selection of
X4 HIV and more rapid disease progression. Approximately 50-60\% of treatment-experienced patients and80-85\% of treatment-naïve patients are infected with the CCR5-tropic virus only. A viral tropism test (Monogram Biosciences, San Francisco, CA, USA) is available to determine the probability of successful treatment, but the cost and turnaround time (3-5 weeks).

In summary, long term maraviroc therapy, meets an unmet need for a well-tolerated drug that reduces viral load with limit side adverse events in a HIV-1 experienced patient with preexisting class resistance.

Current evidence supports the continued development of maraviroc as a potentially useful, alternative treatment for the management of HIV infection. Its favorable toxicity profile makes the drug attractive for consideration in other clinical situations, including patients with earlier stages of disease, cardiovascular risk and viral hepatitis coinfection.

\section{Conflicts of interest}

The authors do not have a commercial or other association that might pose a conflict of interest.

\section{Financial support}

None.

\section{References}

1. Krauskopf L (2007) Pfizer wins U.S. approval for new HIV drug. Reuters.

2. Shilpa S, Homayoon K (2009) Maraviroc: A new CCR5 antagonist. Expert Review of Anti-infective Therapy 7: 9-19

Copyright: $\odot 2019$ Brescini L. This is an open-access article distributed under the terms of the Creative Commons Attribution License, which permits unrestricted use, distribution, and reproduction in any medium, provided the original author and source are credited. 